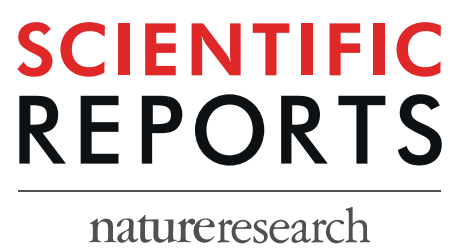

Received: 16 March 2018

Accepted: 19 August 2019

Published online: 04 September 2019

\section{Phospholipase C-related} catalytically inactive protein regulates cytokinesis by protecting phosphatidylinositol 4,5-bisphosphate from metabolism in the cleavage furrow

\author{
Satoshi Asano ${ }^{1}$, Yasuka Ikura ${ }^{1}$, Mitsuki Nishimoto ${ }^{1}$, Yosuke Yamawaki ${ }^{1}$, Kozue Hamao ${ }^{2}$, \\ Keiju Kamijo ${ }^{3}$, Masato Hirata ${ }^{4}$ \& Takashi Kanematsu ${ }^{1,5}$
}

Cytokinesis is initiated by the formation and ingression of the cleavage furrow. Phosphatidylinositol 4,5-bisphosphate $\left[\mathrm{PI}(4,5) \mathrm{P}_{2}\right]$ accumulation followed by RhoA translocation to the cleavage furrow are prerequisites for cytokinesis progression. Here, we investigated whether phospholipase C (PLC)-related

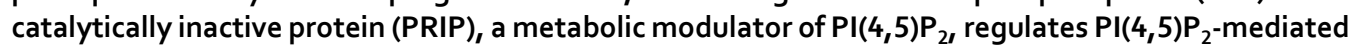
cytokinesis. We found that PRIP localised to the cleavage furrow during cytokinesis. Moreover, HeLa cells with silenced PRIP displayed abnormal cytokinesis. Importantly, $\mathrm{PI}(4,5) \mathrm{P}_{2}$ accumulation at the cleavage furrow, as well as the localisation of RhoA and phospho-myosin II regulatory light chain to the cleavage furrow, were reduced in PRIP-silenced cells. The overexpression of oculocerebrorenal syndrome of Lowe-1 (OCRL1), a phosphatidylinositol-5-phosphatase, in cells decreased $\mathrm{PI}(4,5) \mathrm{P}_{2}$ levels during early cytokinesis and resulted in cytokinesis abnormalities. However, these abnormal cytokinesis phenotypes were ameliorated by the co-expression of PRIP but not by co-expression of a PI(4,5) $\mathrm{P}_{2}$-unbound PRIP mutant. Collectively, our results indicate that PRIP is a component at the cleavage furrow that maintains $\mathrm{PI}(4,5) \mathrm{P}_{2}$ metabolism and regulates RhoA-dependent progression of cytokinesis. Thus, we propose that PRIP regulates phosphoinositide metabolism correctively and mediates normal cytokinesis progression.

Cytokinesis is the final step of cell division, in which a parent cell divides into two daughter cells by the formation and ingression of a cleavage furrow at the plasma membrane after chromosome segregation. A number of phosphoinositides and phosphoinositide-modifying enzymes are involved in cytokinesis, and phosphoinositide production and hydrolysis, which are required for specific steps of cytokinesis, are regulated spatially and temporally ${ }^{1}$. One major phosphoinositide involved is phosphatidylinositol 4,5-bisphosphate $\left[\mathrm{PI}(4,5) \mathrm{P}_{2}\right]$. During cytokinesis, $\mathrm{PI}(4,5) \mathrm{P}_{2}$ accumulates and is maintained at adequate levels at the cleavage furrow from the initiation of furrowing until the completion of constriction in mammalian cells ${ }^{2,3} . \mathrm{PI}(4,5) \mathrm{P}_{2}$ levels in the plasma membrane are regulated by a variety of metabolic enzymes such as phospholipase C (PLC), phosphoinositide kinases,

\footnotetext{
${ }^{1}$ Department of Cellular and Molecular Pharmacology, Division of Basic Life Sciences, Institute of Biomedical and Health Sciences, Hiroshima University, 1-2-3, Kasumi, Minami-ku, Hiroshima, 734-8553, Japan. ${ }^{2}$ Department of Biological Science, Graduate School of Science, Hiroshima University, 1-3-1, Kagamiyama, Higashi-Hiroshima, Hiroshima, 739-8526, Japan. ${ }^{3}$ Division of Anatomy and Cell Biology, Faculty of Medicine, Tohoku Medical and Pharmaceutical University, 1-15-1, Fukumuro, Miyagino-ku, Sendai, Miyagi, 983-8536, Japan. ${ }^{4}$ Oral Medicine Research Center, Fukuoka Dental College, 2-15-1, Tamura, Sawara-ku, Fukuoka, 814-0193, Japan. ${ }^{5}$ Department of Cell Biology and Pharmacology, Faculty of Dental Science, Kyushu University, 3-1-1, Maidashi, Higashi-ku, Fukuoka, 812-8582, Japan. Correspondence and requests for materials should be addressed to T.K. (email: taka-kanematsu@ dent.kyushu-u.ac.jp)
} 
and phosphoinositide phosphatases including oculocerebrorenal syndrome of Lowe-1 (OCRL1), an inositol polyphosphate 5-phosphatase ${ }^{4}$.

Disruption of $\mathrm{PI}(4,5) \mathrm{P}_{2}$ levels at the cleavage furrow causes cytokinesis abnormalities. For example, the overexpression of a kinase-deficient phosphatidylinositol 4-phosphate 5-kinase (PIP5K) mutant and of $\mathrm{PI}(4,5) \mathrm{P}_{2}$-phosphatase synaptojanin or the microinjection of anti-PI(4,5) $\mathrm{P}_{2}$ antibodies impairs cytokinesis in mammalian cells ${ }^{2,5}$. Therefore, properly maintaining $\mathrm{PI}(4,5) \mathrm{P}_{2}$ levels at the cleavage furrow is crucial for normal cytokinesis progression ${ }^{6}$. However, the mechanism by which $\mathrm{PI}(4,5) \mathrm{P}_{2}$ metabolism is regulated during furrow ingression is not fully understood.

Cleavage furrow progression requires the activation of the small GTPase RhoA, which requires epithelial cell transforming sequence 2 (ECT2), a Rho GDP/GTP exchange factor (RhoGEF) ${ }^{7}$. An important role of PI $(4,5) \mathrm{P}_{2}$ is the recruitment of RhoA to the target membrane, as the depletion of $\mathrm{PI}(4,5) \mathrm{P}_{2}$ inhibits the recruitment of RhoA to the cleavage furrow in cytokinesis ${ }^{3,8,9}$. RhoA has a positively charged amino acid cluster at the carboxy-terminus, which can directly interact with negatively charged phosphoinositides, including $\mathrm{PI}(4,5) \mathrm{P}_{2}$, in vitro ${ }^{9,10}$. ECT2 also interacts with phosphoinositides, including $\mathrm{PI}(4,5) \mathrm{P}_{2}$, in vitro ${ }^{11}$. Therefore, the direct binding of Rho-GEF and RhoA to $\mathrm{PI}(4,5) \mathrm{P}_{2}$ in the cleavage furrow could be an important process in cytokinesis progression.

Actin filaments and myosin-II are evolutionarily conserved force-generating components of the contractile ring during cytokinesis. Contractile force is generated by non-muscle myosin II, which is regulated by RhoA/ Rho-associated protein kinase (ROCK) signalling. Activated ROCK phosphorylates myosin II regulatory light chain (MRLC) directly and also phosphorylates myosin phosphatase-targeting subunit 1, inactivating it. Consequently, phospho-MRLC (Ser19) is augmented ${ }^{12}$, which enhances the ATPase activity of myosin II and promotes the assembly of monomers of myosin II into bipolar filaments, followed by the generation of a contractile force, which then cleaves the parent cell into two daughter cells ${ }^{13,14}$.

PLC-related catalytically inactive protein (PRIP) is a unique protein with high homology to the PLC $\delta 1$ isozyme but lacking PLC activity ${ }^{15-17}$. There are two isoforms of PRIP in mammals, PRIP1 and PRIP2: the former is expressed mainly in the brain and the lung, and the latter is expressed ubiquitously ${ }^{18,19}$. PRIP1 was originally isolated as a cytosolic protein containing a pleckstrin homology $(\mathrm{PH})$ domain, which binds to inositol-1,4,5-triphosphate $\left[\operatorname{Ins}(1,4,5) \mathrm{P}_{3}\right]^{16,20,21}$. Subsequently, PRIP1 and PRIP2 were reported to bind to $\mathrm{PI}(4,5) \mathrm{P}_{2}$ via its $\mathrm{PH}$ domain and localise to the plasma membrane ${ }^{22-24}$. Although the sequence of the PRIP PH domain is similar to that of PLC $\delta 1$, PRIPs exhibit distinct functional characteristics with respect to $\operatorname{Ins}(1,4,5) \mathrm{P}_{3}$ and $\mathrm{PI}(4,5) \mathrm{P}_{2}$ binding ${ }^{25}$. Amino acid R40 in PLC $\delta \mathrm{PH}$ is a critical amino acid residue for Ins $(1,4,5) \mathrm{P}_{3}$ binding, and PRIP1 $\mathrm{PH}(\mathrm{R} 134 \mathrm{Q})$ mutant (R134 corresponds to R40 in $\mathrm{PLC} \delta \mathrm{PH})$ fails to bind with $\operatorname{Ins}(1,4,5) \mathrm{P}_{3}$ or $\mathrm{PI}(4,5) \mathrm{P}_{2}^{23,26}$.

We recently reported that PRIP negatively regulates the conversion of $\mathrm{PI}(4,5) \mathrm{P}_{2}$ into phosphatidylinositol 3,4,5-trisphosphate by phosphoinositide 3-kinase (PI3K), a process that suppresses the migration of cancer cells ${ }^{27}$. However, little is known regarding the involvement of PRIP in $\mathrm{PI}(4,5) \mathrm{P}_{2}$-dependent cytokinetic events. Therefore, we examined the potential role and regulatory mechanism of PRIP in cytokinesis.

\section{Results}

PRIP accumulates at the cleavage furrow during cytokinesis. We first investigated the localisation of PRIP during cytokinesis using HeLa and HEK293 cells transiently transfected with PRIP1 or PRIP2. Both enhanced green fluorescent protein (EGFP)-PRIP1 and EGFP-PRIP2 signals in HeLa cells clearly localised to the cleavage furrow, where phosphorylated MRLC signal (pMRLC; used as a cleavage furrow marker) was observed (Fig. 1a). Similar results were observed in HEK293 cells, in which EGFP signals of PRIP1 and PRIP2 accumulated at the cleavage furrow with pMRLC signal. Some PRIP1 and PRIP2 signals were also observed on the plasma membrane surrounding HEK293 cells, whereas control EGFP-vector signal was observed in the cytoplasm (Fig. 1a).

PRIP1 was originally purified from a cytosolic fraction but has subsequently been purified from a membrane fraction ${ }^{20,24}$. Therefore, we next examined the localisation of PRIP1 and PRIP2 during interphase. EGFP-PRIP1 and EGFP-PIRP2 mainly localised to the cytoplasm, with some signal being observed on the plasma membrane in both HEK293 and HeLa cells (Fig. 1b). These observations suggest that PRIP gravitates toward a component of the cleavage furrow during cytokinesis.

PRIP participates in the formation and ingression of the cleavage furrow. To examine the role of PRIP at the cleavage furrow, we investigated the cytokinesis machinery regulated by PRIP. We used three cell types in this study: HeLa (a human cervical cancer cell line), HEK293 (a human embryonic kidney cell line), and MCF-7 (a human breast cancer cell line). PRIP2 mRNA expression was substantial in HeLa and HEK293 cells but minimal in MCF-7 cells according to reverse transcription-PCR analysis. In contrast, PRIP1 mRNA expression was substantial in HEK293 cells but minimal in HeLa and MCF-7 cells (Supplementary Fig. S1).

Endogenous PRIP2 was successfully depleted from HeLa cells, in which PRIP1 is not expressed, resulting in levels of about $40 \%$ and $10 \%$ that in control cells following transient transfection with PRIP2-specific siRNAs PRIP2-si1 and PRIP2-si2, respectively (Fig. 2a). PRIP2 participation in cell division was analysed using a live-cell imaging technique. HeLa cells transfected with control siRNA displayed normal cell division (Fig. 2b, control), and almost all control HeLa cells underwent cytokinesis within $3 \mathrm{~h}$ of synchronisation with and release from monastrol, a potent cell-permeable mitosis inhibitor (Fig. 2c, control). The peak of cytokinesis onset occurred at 61-90 min after release from monastrol (Fig. 2d, control siRNA). By contrast, depletion of PRIP2 by PRIP2-si2 in HeLa cells retarded the onset of cytokinesis, with no substantial peak of onset apparent during 31-180 min (Fig. 2d). Approximately $60 \%$ of PRIP2-si2-transfected cells underwent initiation of cytokinesis within $3 \mathrm{~h}$ after release from monastrol (Fig. 2c) and showed normal cell division (Fig. 2b, normal in PRIP2-si2). However, the remaining $40 \%$ of cells displayed abnormal cytokinesis and failed to undergo cytokinesis (Fig. 2c). These abnormal phenotypes were classified into no furrowing, abnormal furrowing, and regression (Fig. $2 \mathrm{~b}$ ). 
a

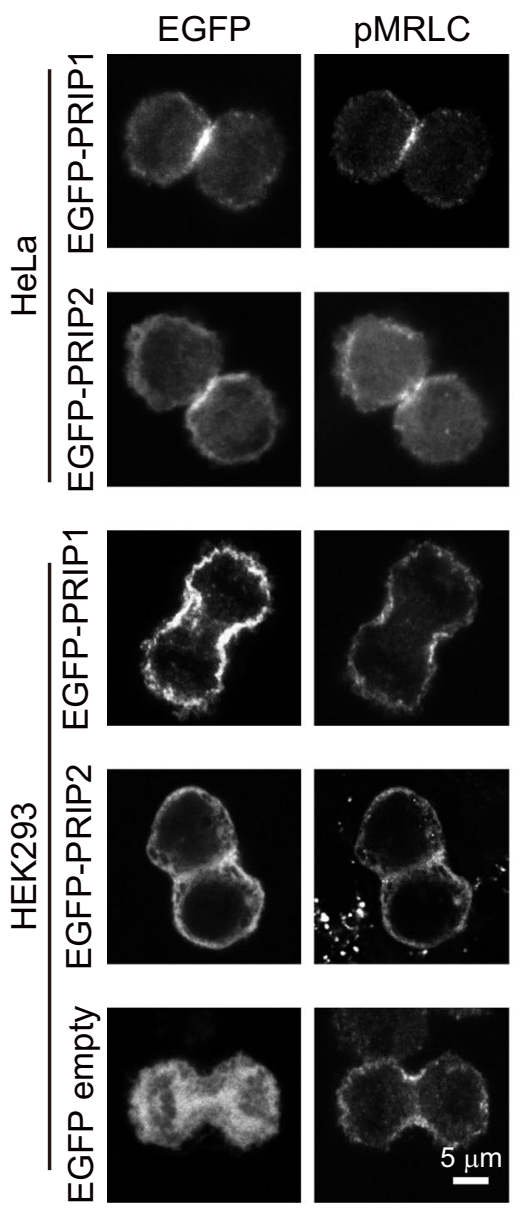

b
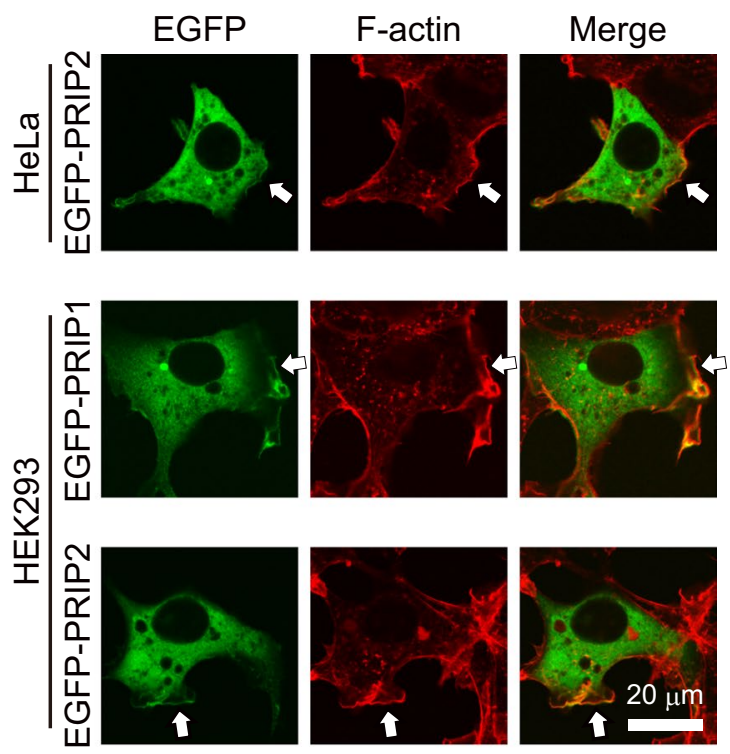

Figure 1. Accumulation of PRIP at the cleavage furrow during cytokinesis. (a,b) EGFP-PRIP signals were detected in HeLa and HEK293 cells during cytokinesis (a) and interphase (b). pMRLC (a) and F-actin (b) were stained with an anti-phospho-MRLC antibody followed by an Alexa Fluor 594-conjugated secondary antibody and ActinRed 555 ReadyProbes reagent, respectively. Images were obtained by confocal microscopy. Arrows in (b) show the presence of PRIP on F-actin on the inner face of the plasma membrane. Similar images were satisfactorily obtained from more than three independent experiments.

We then analysed the duration of cytokinesis. Dividing HeLa cells exhibited delayed progression of furrow ingression in PRIP2 siRNA-transfected cells compared with that of controls (Fig. 2e, control/empty vs. PRIP-si2/ empty). A plot of cytokinesis speed, which was determined by the rate of change in furrow length, was shifted to the right in cells transfected with PRIP-si2/empty EGFP-vector compared with that in cells transfected with control siRNA/empty EGFP-vector (Fig. 2f). However, this delay was rescued by transfection with EGFP-Prip1 in PRIP2-si2-treated HeLa cells (Fig. 2e,f). The time to completion of cytokinesis was similar among control siRNA-transfected HeLa cells and HeLa cells overexpressing exogenous Prip1 or PRIP2 (Fig. 2e, control siRNA-transfected experiments in the upper and lower panels, and Fig. 2g). These data indicate that PRIP overexpression does not affect cytokinesis in HeLa cells; however, PRIP2 gene depletion inhibits cytokinesis progression, which is ameliorated by the exogenous expression of PRIP (Fig. $2 \mathrm{~h}$ ).

PRIP regulates MRLC phosphorylation at the cleavage furrow during cytokinesis. An actomyosin-based contractile ring is present at the cell equator. Actin filaments and non-muscle myosin II are components of the contractile ring during cytokinesis, and these generate the constricting force. The phosphorylation of MRLC at Ser 19 activates the ATPase activity of myosin II, which promotes binding to and motility along actin filaments ${ }^{28}$. To investigate whether PRIP affects the localisation of pMRLC to the cleavage furrow, we performed immunocytochemistry with an anti-pMRLC antibody and stained F-actin with phalloidin. pMRLC signal was detected at the cleavage furrow in control siRNA-transfected HeLa cells during the initiation phase and the early and late stages of furrow ingression (Fig. 3a, upper panels, control siRNA). However, transfection of HeLa cells with PRIP2 siRNA reduced pMRLC and F-actin signals at the cleavage furrow compared with those in the controls (Fig. 3a, upper vs. middle panels). In experiments involving co-transfection with a full-length Prip1 gene and PRIP2 siRNA, EGFP-PRIP1 localised at the cleavage furrow and rescued the localisation of pMRLC to the furrow (Fig. 3a, middle vs. lower panels). Next, the signal intensities of pMRLC and F-actin at the cleavage 
b

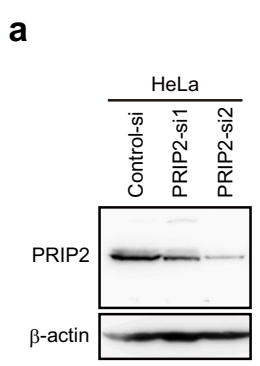

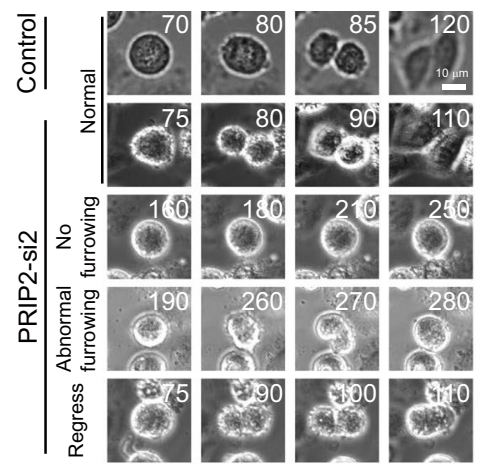

C

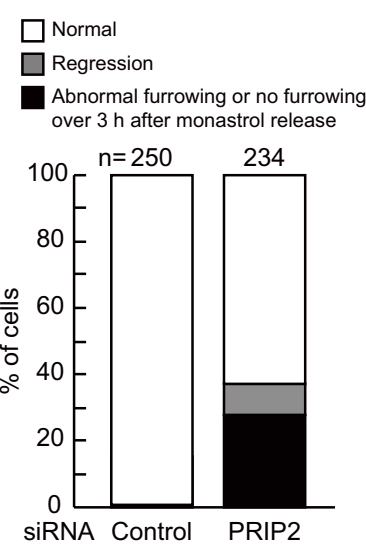

d

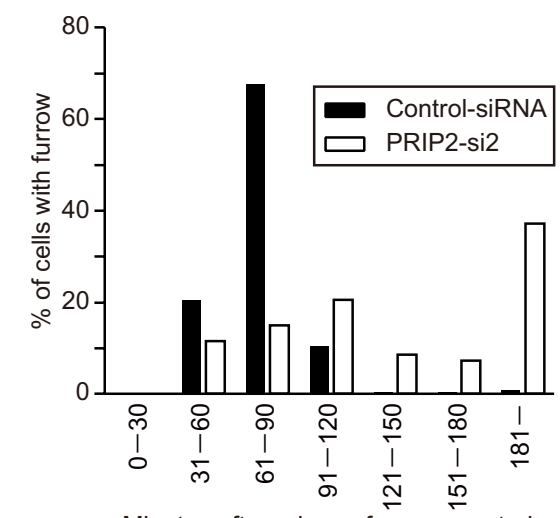

e

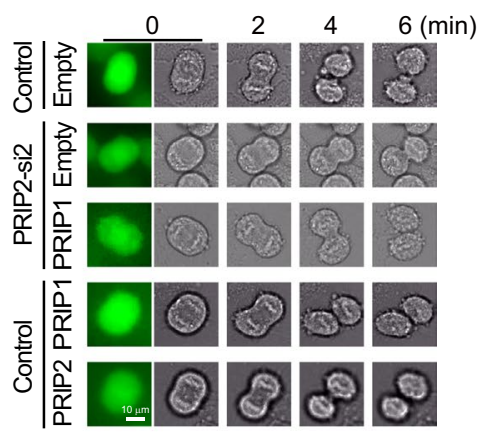

Minutes after release from monastrol

$\mathbf{f}$

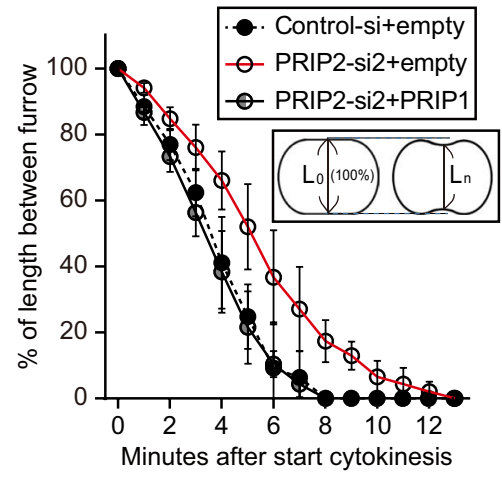

g

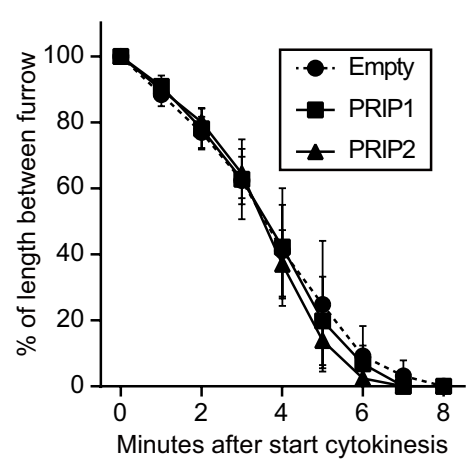

h

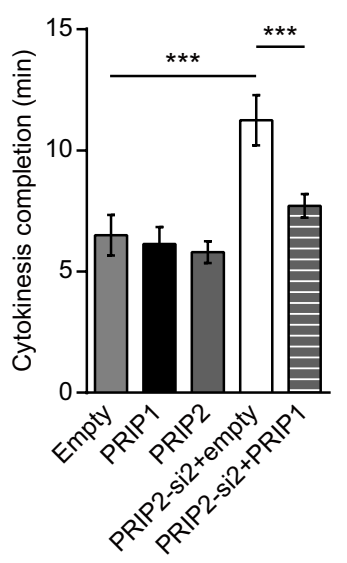

Figure 2. PRIP participates in the formation and ingression of the cleavage furrow. (a) Success of PRIP2 silencing in HeLa cells analysed by western blotting using the indicated antibodies. $\beta$-actin was used as a loading control. Control siRNA (Control-si) and PRIP2 siRNAs (PRIP2-si1 and PRIP2-si2) were used. Each of the original blots is shown in Supplementary Fig. S7a,b. (b-d) A time course analysis of dividing HeLa cells via time-lapse image analyses. The experiments were repeated at least three times, and a set of representative time-lapse images is shown in (b). The Arabic numerals in (b) indicate times (min) after the removal of monastrol from culture media. The frequency of the type of cytokinesis failure (normal: a dividing cell; regression: a cell starting a furrow ingression but not undergoing complete cytokinesis; abnormal furrowing: a cell having asymmetric furrow formation and ingression in the midzone) was analysed in over $230 \mathrm{HeLa}$ cells (c). The distribution of cytokinesis onset time is shown in (d). The y axis of the graph indicates the percentage of cells with furrow measured at each time interval divided by the total mitotic cell number. (e-h) Aberration of cytokinesis in PRIP-silenced HeLa cells and restoration by PRIP gene transfection. Cells were transfected with the indicated siRNAs together with EGFP vector (empty), EGFP-Prip1 (PRIP1), or EGFPPRIP2 (PRIP2). Representative time-lapse series of fluorescence images during furrow ingression are shown (e). The experiments were repeated at least three times. Data are presented as relative furrow ingression [see 
the schematic diagram in (f)]. The y axis of the graphs in (f) and (g) indicates the percentage of distance $\left(\mathrm{L}_{\mathrm{n}}\right)$ between the two constricting poles measured at each time point divided by initial distance $\left(\mathrm{L}_{0}\right)$ at the beginning of cytokinesis. Mean time to the completion of furrowing in (f,g) is shown in (h). The data are presented as the means \pm SD ( $\mathrm{n}>20$ for each group). $* * * p<0.001$ (Kruskal-Wallis test followed by Dunn's multiple comparison test).

furrow were assessed by dividing each furrow intensity by the corresponding total cell intensity in the early stage of furrow ingression (Fig. 3b). The fluorescence intensities of the two signals in PRIP2-silenced cells were restored by Prip1 co-transfection.

The pan MRLC signal localised on the plasma membrane in control cells during cytokinesis, and this was not altered in PRIP2-silenced HeLa cells (Fig. 3c). Moreover, the pan MRLC signal at the cleavage furrow did not differ between control siRNA and PRIP2 siRNA groups (Fig. 3d). These data suggest that PRIP participates in the phosphorylation of MRLC at the cleavage furrow without affecting MRLC accumulation at the furrow.

A phospho-mimic MRLC mutant rescues delayed cytokinesis progression in PRIP-silenced HeLa cells. To verify that PRIP participates in pMRLC-mediated cytokinesis progression, we used two MRLC mutants, a phospho-mimic MRLC (AD-MRLC; amino acids Thr18 and Ser19 substituted with Ala and Asp, respectively) and a non-phosphorylatable form of MRLC (AA-MRLC; both Thr18 and Ser19 are substituted with Ala). EGFP-AA-MRLC localised to the cleavage furrow in HeLa cells (Supplementary Fig. S2), suggesting that localisation of MRLC to the cleavage furrow does not require Thr18/Ser19 phosphorylation. It was previously reported that transfection with exogenous AA-MRLC inhibited the phosphorylation of endogenous MRLC at the cleavage furrow ${ }^{29}$. We then analysed cytokinesis speed in normal HeLa cells and found that it was decreased in $A A-M R L C$-overexpressing cells, whereas it was similar in $A D$ - $M R L C$-overexpressing and empty vector-transfected controls (Fig. 4a), suggesting that reducing the phosphorylation of MRLC inhibits cytokinesis speed.

We next examined whether the AA-MRLC and AD-MRLC mutants affect cytokinesis progression modulated by PRIP. The decrease in cytokinesis speed in PRIP2-depleted HeLa cells was not rescued by transfection with $A A-M R L C$. However, cytokinesis speed was restored to control siRNA-transfected cell levels upon transfection with $A D-M R L C$ (Fig. 4b-d), indicating that PRIP modulates the upstream signalling of MRLC phosphorylation.

PRIP is involved in RhoA-mediated cytokinesis. Rho catalyses actin nucleation and polymerisation to form actin filaments and activates myosin cross-linking ${ }^{30}$. Downstream of Rho activation, RhoA induces mammalian homologue of Drosophila diaphanous ( $\mathrm{mDia}$ )-mediated actin nucleation and polymerisation, as well as ROCK/pMRLC-dependent myosin II activation, consequently leading to the assembly and contraction of the actomyosin ring at the cleavage furrow ${ }^{31,32}$. PRIP2-knockdown HeLa cells exhibited a decrease in the thickness of F-actin as well as a reduced signal intensity of pMRLC in the cleavage furrow (Fig. 3a,b). Therefore, we wondered whether PRIP regulates the signalling of RhoA as an upstream regulator in cytokinesis progression. To this end, we investigated RhoA localisation at the cleavage furrow by immunocytochemistry with an anti-RhoA antibody. The RhoA signal was weaker at the cleavage furrows of both PRIP2-si1- and PRIP2-si2-transfected HeLa cells than that in control siRNA-transfected cells during the initiation and early steps of cytokinesis (Fig. 5a). In addition, we detected the aberrant localisation of RhoA in PRIP2-silenced cells (Fig. 5a, arrowheads). PRIP2-silenced cells showed a significant decrease in the fluorescence intensity of RhoA signal at the cleavage furrow (Fig. 5b). These results suggest that PRIP is necessary for the proper localisation of RhoA at the cleavage furrow.

PRIP coordinates $\mathrm{PI}(\mathbf{4}, \mathbf{5}) \mathrm{P}_{2}$ levels at the cleavage furrow. $\mathrm{PI}(4,5) \mathrm{P}_{2}$ is necessary for correct furrow ingression during cytokinesi ${ }^{5,8}$. Rho1, the budding yeast RhoA homologue, binds to $\mathrm{PI}(4,5) \mathrm{P}_{2}$ directly, which is required to complete cytokinesis ${ }^{9}$. In addition, $\mathrm{PI}(4,5) \mathrm{P}_{2}$ regulates the accumulation of RhoA at the cleavage furrow in HeLa cells ${ }^{3}$. We previously reported a protective effect of PRIP on PI $(4,5) \mathrm{P}_{2}$ metabolism in migrating cells $\mathrm{s}^{27}$. Therefore, we predicted that $\mathrm{PI}(4,5) \mathrm{P}_{2}$ accumulation at the cleavage furrow would be regulated by PRIP. To directly monitor plasma membrane $\mathrm{PI}(4,5) \mathrm{P}_{2}$ levels during cytokinesis, we successfully established a HEK293 cell line that stably expressed the EGFP-tagged PH domain of PLC 1 (EGFP-PLC $\delta \mathrm{PH})$, a probe for $\mathrm{PI}(4,5) \mathrm{P}_{2}$. The isolated cell line had a single peak distribution of EGFP intensity, as determined by flow cytometry (Fig. 5c), and EGFP-PLC 8 PH signal was uniformly detected at the plasma membrane in trypsinised cells (Fig. 5d). Endogenous PRIP2 in the established HEK293 cells was successfully depleted to levels of about 40\% and $20 \%$ by transfection with PRIP2-sil and PRIP2-si2, respectively (Supplementary Fig. S3). PRIP2 silencing dispersed the EGFP-PLCSPH signal from the cleavage furrow to the cytoplasm (Fig. 5e, left vs. middle panels, and Fig. 5f). However, upon co-transfection of Halo-PRIP1 in PRIP2-silenced cells, Halo-PRIP1 signal was highly retained at the cleavage furrow, together with EGFP-PLCSPH signal (Fig. 5e, right panels, and Fig. 5f).

To further investigate the relationship between PRIP localisation at the cleavage furrow and PRIP binding to $\mathrm{PI}(4,5) \mathrm{P}_{2}$, BODIPY FL-PI $(4,5) \mathrm{P}_{2}$ was exogenously added to HeLa cell culture before examination of PRIP localisation. BODIPY FL-PI $(4,5) \mathrm{P}_{2}$ signal was observed at the cleavage furrow and the plasma membrane, and in intracellular components (Supplementary Fig. S4). Consistently, DsRed2-PRIP1 and DsRed2-PRIP2 signals were co-localised with BODIPY FL-PI $(4,5) \mathrm{P}_{2}$ signal, including at the cleavage furrow. However, the signal of DsRed2-PRIP1(R134Q), in which the PH domain fails to bind $\mathrm{PI}(4,5) \mathrm{P}_{2}{ }^{33}$, was observed in the cytoplasm and failed to accumulate at the cleavage furrow (Supplementary Fig. S4, panels on the far right). These results suggest that PRIP arrives to the cleavage furrow and is interdependently retained with $\mathrm{PI}(4,5) \mathrm{P}_{2}$ in this location via the PH domain of PRIP. 
a
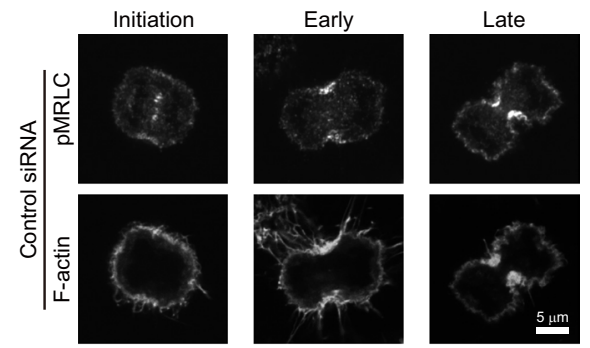

b
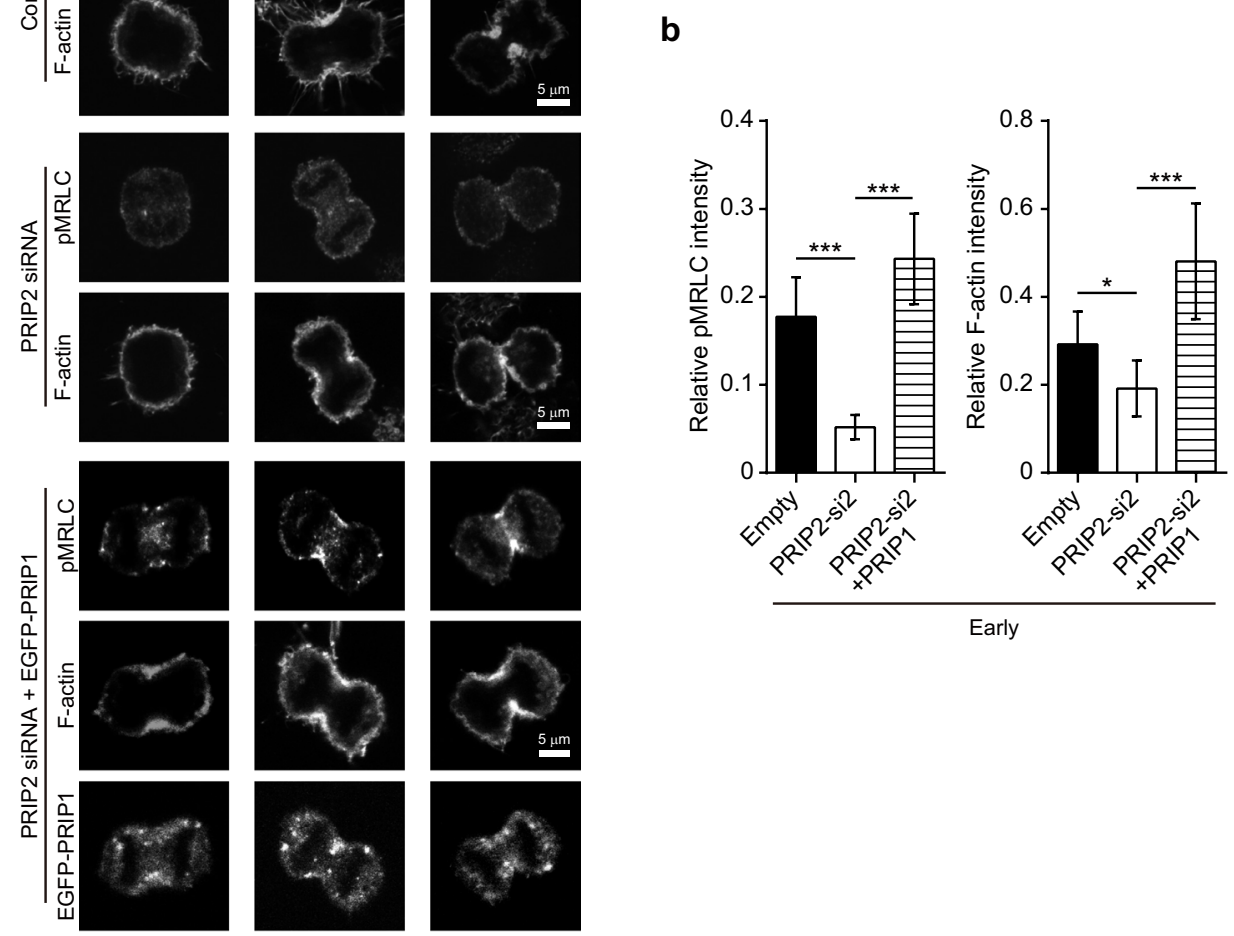

C
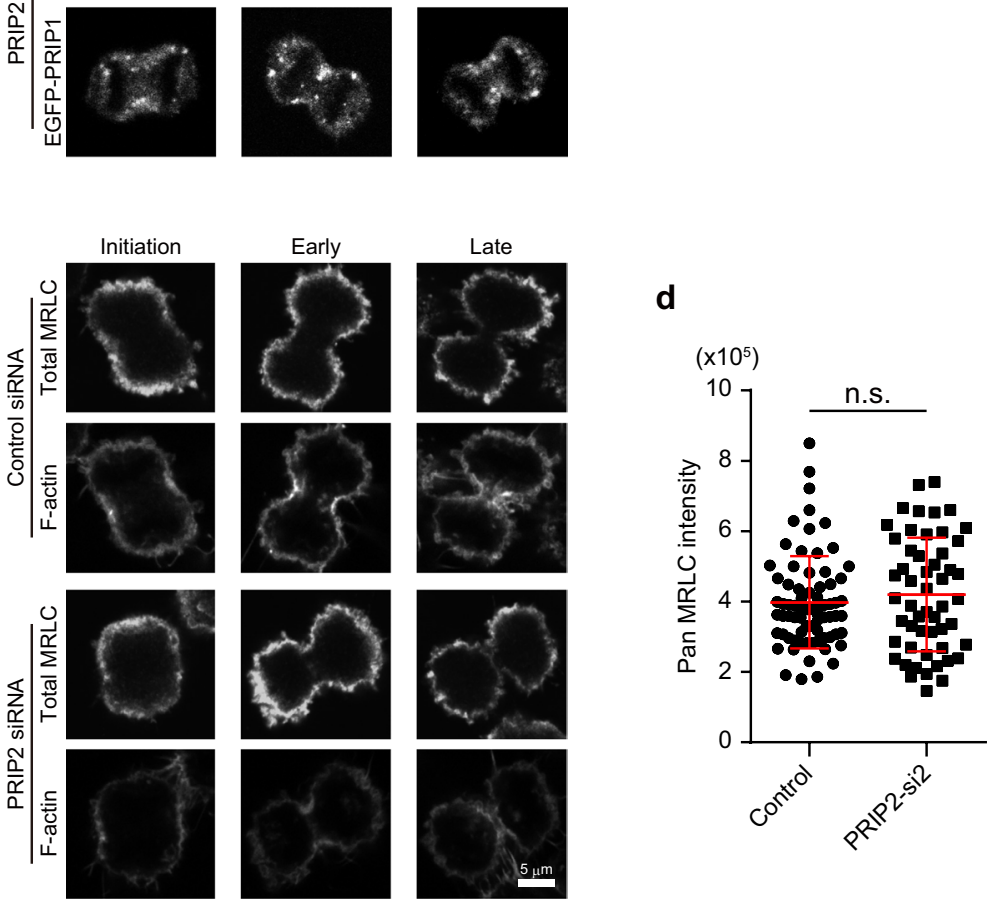

Figure 3. PRIP regulates MRLC phosphorylation at the cleavage furrow during cytokinesis. (a-d) HeLa cells were transfected with a control siRNA (a,c, upper panels), PRIP2-si2 (a, middle panels; c, lower panels), or PRIP2-si2 together with EGFP-tagged Prip1 (a, lower panels). Phosphorylated MRLC (pMRLC) (a), pan MRLC (c), and F-actin $(\mathbf{a}, \mathbf{c})$ were stained with an anti-phospho-MRLC antibody, anti-total MRLC antibody, and Alexa Fluor 350-labelled phalloidin, respectively. The images were obtained by confocal microscopy. Representative set of images is shown during cytokinesis [initiation phase, and early and late stages in furrow ingression] in (a,c). Graphs in (b) show the relative fluorescence intensity of pMRLC (left panel) and F-actin (right panel) at the cleavage furrow in an early stage of furrow ingression. The relative fluorescence intensity (y axis) was defined as the fluorescence intensity of pMRLC or F-actin in the cleavage furrow divided by the total intensity of pMRLC or F-actin in the cell, respectively. The data are presented as the mean $\pm S D$ ( $n=46$ for each group). ${ }^{*} p<0.05$, *** $p<0.001$ (Kruskal-Wallis test followed by Dunn's multiple comparison test). Graph in (d) shows the fluorescence intensity (arbitrary units) of pan MRLC at the cleavage furrow. The data are presented as the means $\pm S D$ ( $n>60$ for each group). n.s.: not significant (Student's $t$-test). 


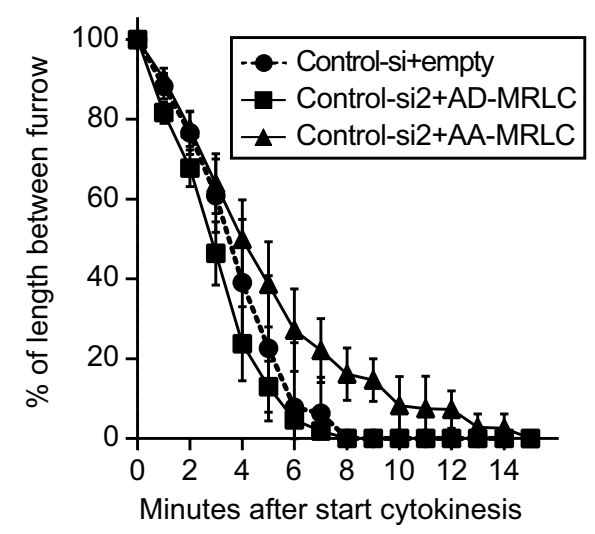

C

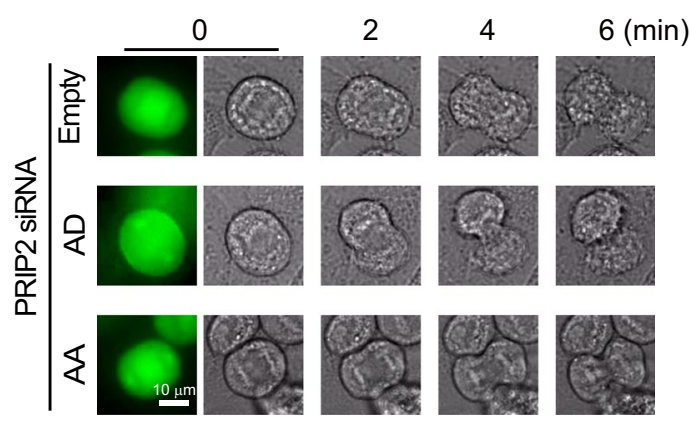

b

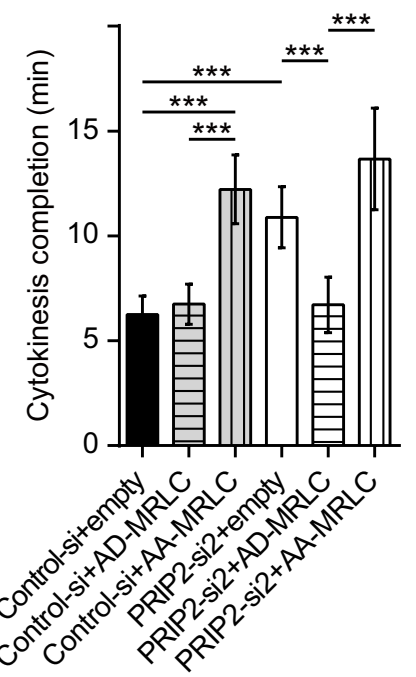

d

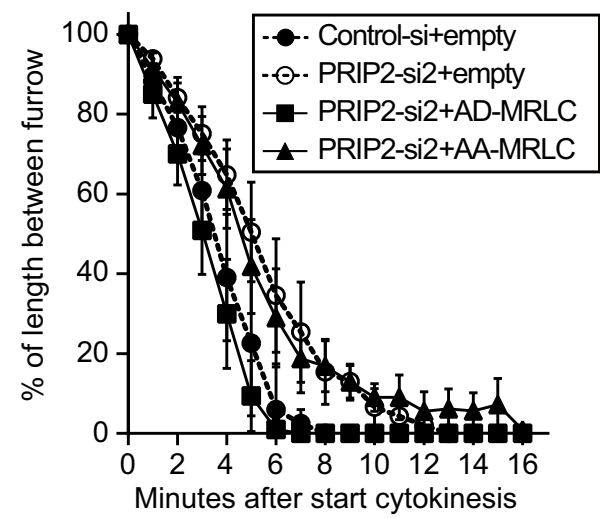

Figure 4. A phospho-mimic MRLC mutant restores delayed cytokinesis progression in PRIP-silenced HeLa cells. (a-d) Progression of furrow ingression was observed in HeLa cells transfected with the indicated siRNAs together with EGFP vector (empty), EGFP-AD-MRLC (AD-MRLC), or EGFP-AA-MRLC (AA-MRLC). The polygonal line graphs in (a,d) show calculation results; i.e., the measured width of the cleavage furrow divided by the initial width $(0 \mathrm{~min})$. The bar graphs in (b) represent the mean time to completion of furrowing in (a,d). A set of representative time-lapse images is shown in (c). Each experiment was performed at least three times (a-d), and more than twenty images displaying similar features were obtained (c). The data are presented as the means $\pm S D[n \geq 20$ for each group $(\mathbf{a}, \mathbf{b}, \mathbf{d})]$. $* * * p<0.001$ (Kruskal-Wallis test followed by Dunn's multiple comparison test).

PRIP maintains $\mathrm{PI}(4,5) \mathrm{P}_{2}$ at the cleavage furrow during cytokinesis. Although PRIP is engaged in maintaining $\mathrm{PI}(4,5) \mathrm{P}_{2}$ levels in the cleavage furrow (Fig. 5f), it remains unknown how PRIP regulates $\mathrm{PI}(4,5) \mathrm{P}_{2}$ levels. We hypothesised that the mechanism by which PRIP regulates $\mathrm{PI}(4,5) \mathrm{P}_{2}$ levels at the cleavage furrow is either via the upregulation of new $\mathrm{PI}(4,5) \mathrm{P}_{2}$ synthesis or the downregulation of $\mathrm{PI}(4,5) \mathrm{P}_{2}$ metabolism into other phospholipids. To address this issue, we examined the turnover of $\mathrm{PI}(4,5) \mathrm{P}_{2}$ at the cleavage furrow using stably EGFP-PLC8PH-expressing HEK293 cells. The synthesis and metabolism rates of PI(4,5) $\mathrm{P}_{2}$ were assessed by measuring the GFP intensity of PLCSPH via fluorescence recovery after photobleaching (FRAP) analysis. We selected a cell starting cytokinesis and bleached the region of the cleavage furrow with a laser line. At the non-bleached side of the cleavage furrow, the GFP intensity did not differ between control siRNA- and PRIP2-si1-transfected cells (Fig. 6a,b), suggesting that the $\mathrm{PI}(4,5) \mathrm{P}_{2}$ synthesis rate is similar between the two types of cells. By contrast, on the bleached side, the recovery of GFP intensity was slower in PRIP-expressing control HEK293 cells than in PRIP2-silenced cells (Fig. 6a,c), suggesting that PRIP maintains $\mathrm{PI}(4,5) \mathrm{P}_{2}$ at the cleavage furrow and suppresses $\mathrm{PI}(4,5) \mathrm{P}_{2}$ metabolism.

It is reported that silencing of OCRL1 (an inositol polyphosphate 5-phosphatase) in HeLa cells results in the accumulation of $\mathrm{PI}(4,5) \mathrm{P}_{2}$ at the intercellular bridge in late cytokinesis ${ }^{34}$. We ensured that Halo-OCRL1 overexpression efficiently reduced $\mathrm{PI}(4,5) \mathrm{P}_{2}$ levels at the intercellular bridge compared with that in control cells in late cytokinesis (Supplementary Fig. S5). Then, we used OCRL1-overexpressing cells to investigate whether PRIP modulates $\mathrm{PI}(4,5) \mathrm{P}_{2}$ metabolism by OCRL1 at the cleavage furrow. We found that, during cleavage 
a
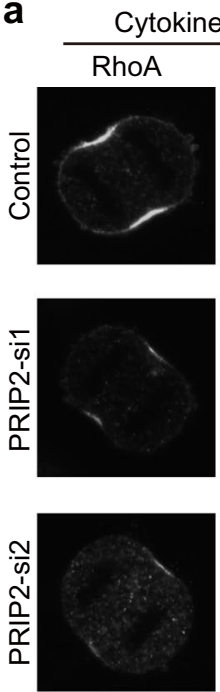

C

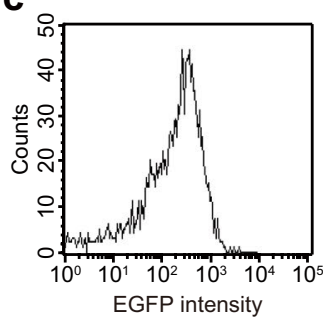

d

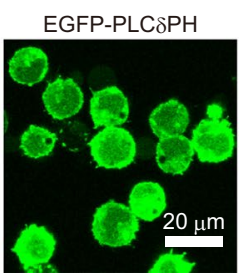

Early cytokinesis
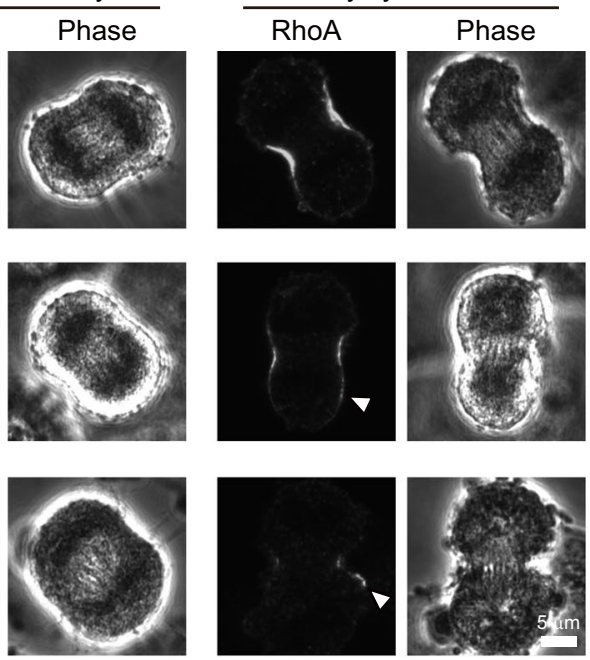

e
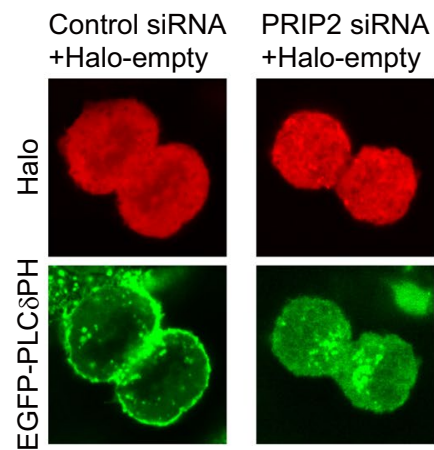

PRIP2 siRNA +Halo-PRIP1
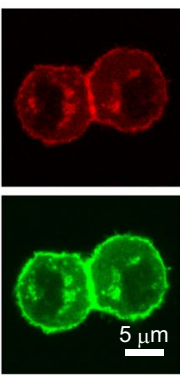

b

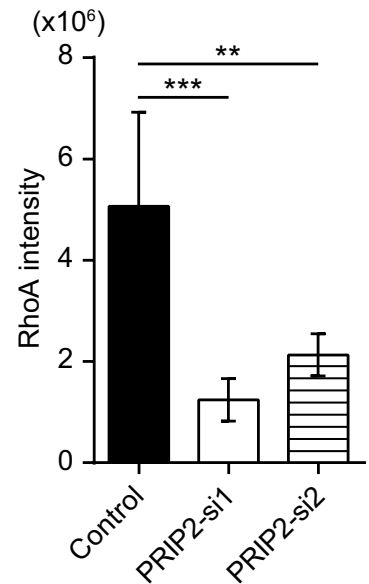

f

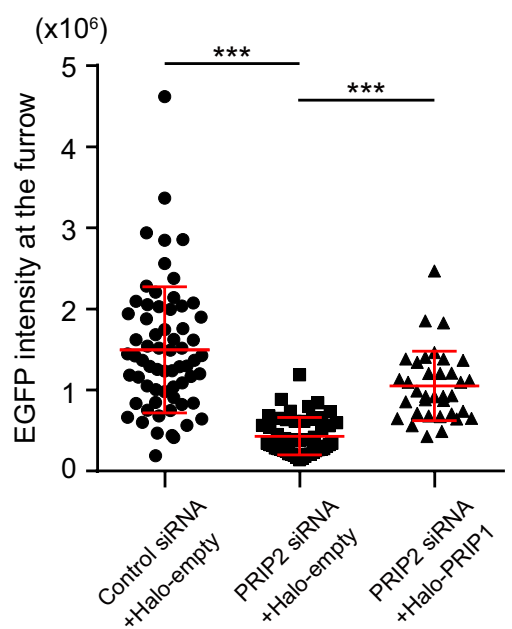

Figure 5. PRIP regulates the accumulation of RhoA and coordinates $\mathrm{PI}(4,5) \mathrm{P}_{2}$ levels at the cleavage furrow. (a,b) Transfection with PRIP2-si1 or PRIP2-si2 inhibits RhoA accumulation at the cleavage furrow in HeLa cells. Representative images in a stage of cytokinesis (cytokinesis entry and early cytokinesis) are shown in (a). RhoA signals were visualised using a specific antibody. Arrowheads in (a) indicate abnormal localisation of RhoA. Graph in (b) shows the fluorescence intensity (arbitrary units) of RhoA at the cleavage furrow in early cytokinesis. The data are presented as the mean $\pm \mathrm{SD}(\mathrm{n}=45$ for each group $) . * * p<0.01$, *** $p<0.001$ (Kruskal-Wallis test followed by Dunn's multiple comparison test). (c-f) PRIP2 silencing inhibits $\mathrm{PI}(4,5) \mathrm{P}_{2}$ accumulation at the cleavage furrow in HEK293 cells. HEK293 cells stably expressing EGFP-tagged pleckstrin homology domain of PLC 1 (EGFP-PLC $8 \mathrm{PH})$ were transfected with the indicated siRNAs (e,f) together with Halo-tagged vector (Halo-empty) or Halo-tagged PRIP1 (Halo-PRIP1) (e,f). Analyses of EGFP intensity of the cells were performed by flow cytometry (c). The cells were trypsinised and observed (d). (e,f) Representative images of EGFP-PLC $\delta \mathrm{PH}$ and Halo-tagged proteins in a stage of cytokinesis are shown in (e). Graph in (f) presents the mean intensity of EGFP in the cleavage furrow \pm SD ( $\mathrm{n}>60$ for each group). $* * * p<0.001$ (Kruskal-Wallis test followed by Dunn's multiple comparison test).

furrow formation, exogenously expressed Halo-OCRL1 was diffusely distributed in HEK293 cells, and PI(4,5) $\mathrm{P}_{2}$ levels (PLC $8 \mathrm{PH}$ signal) were obviously reduced at the plasma membrane, including the cleavage furrow, compared with that in Halo vector-transfected control cells (Fig. 6d, compare first two panels). Prip1 and Halo-OCRL1-co-transfected HEK293 cells exhibited the accumulation of PLC8PH signal with DsRed-PRIP1 signal (Fig. 6d, third panel). However, DsRed-Prip1(R134Q)-mutant and Halo-OCRL1-co-transfected HEK293 cells exhibited blurred signals of PLC $\delta$ PH and DsRed-PRIP1(R134Q) (Fig. 6d, far right panel). The PI(4,5) $\mathrm{P}_{2}$ levels at the cleavage furrow were then assessed based on the intensity of the PLC $\delta \mathrm{PH}$ signal. Prip1 overexpression in Halo-OCRL1-transfected HEK293 cells restored PI $(4,5) \mathrm{P}_{2}$ levels to those in non-Halo-OCRL1-transfected cells, but Prip1(R134Q) overexpression did not (Fig. 6e).

To investigate the effects of PRIP in OCRL1-expressed cells on cytokinesis progression, we performed live-cell imaging. We used MCF-7 cells stably expressing Prip1, Prip1(R134Q), or EGFP vector ${ }^{27}$ in which 
a
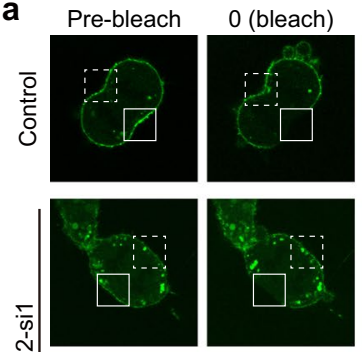

$\frac{\bar{\alpha}}{\alpha}$
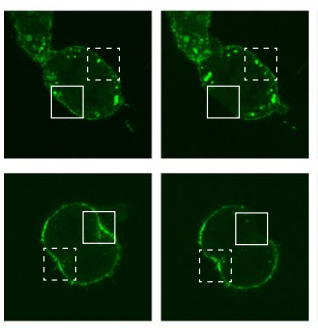

d
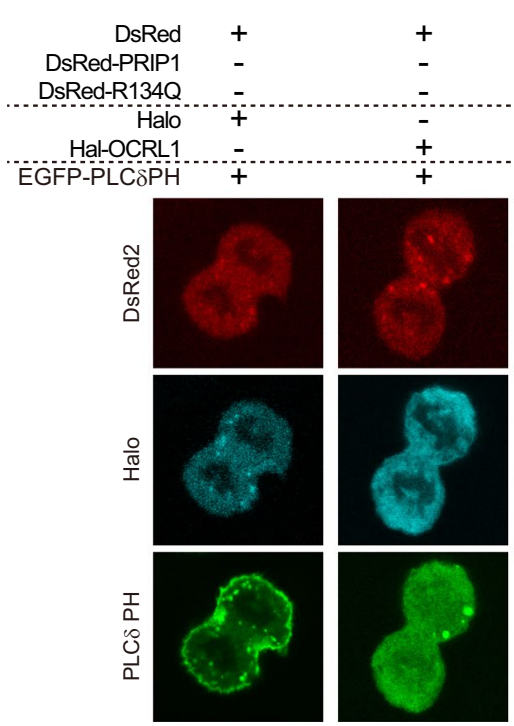

e

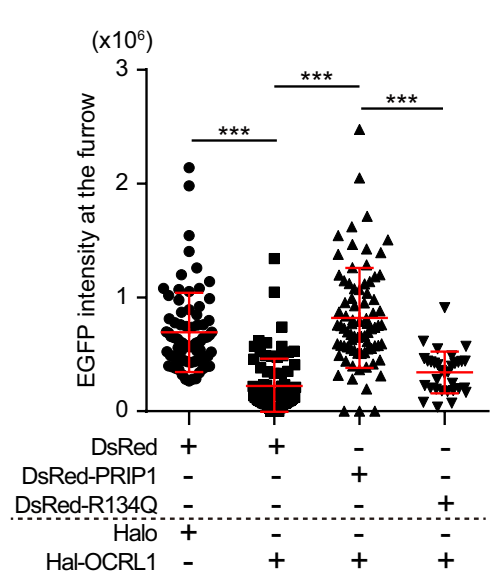

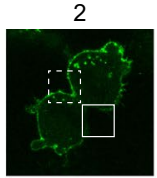
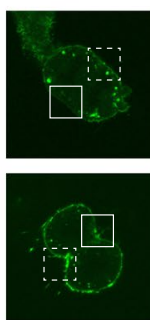

4
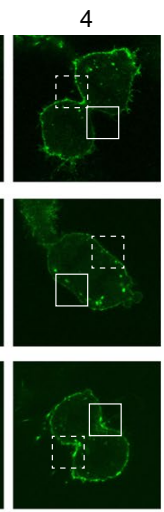
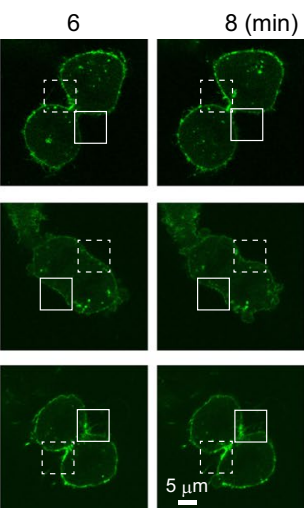
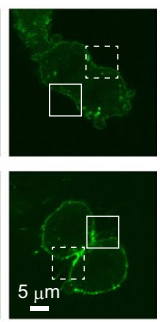

$5 \underline{\mu m}$

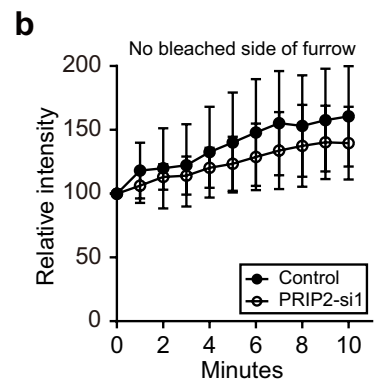

C

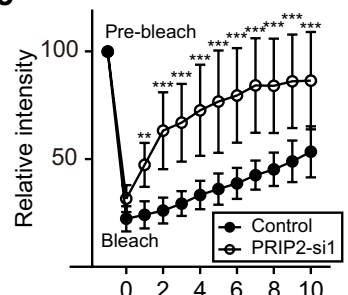

Minutes after photo-bleaching
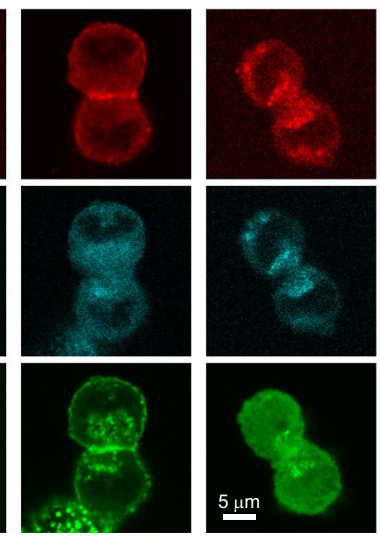

f

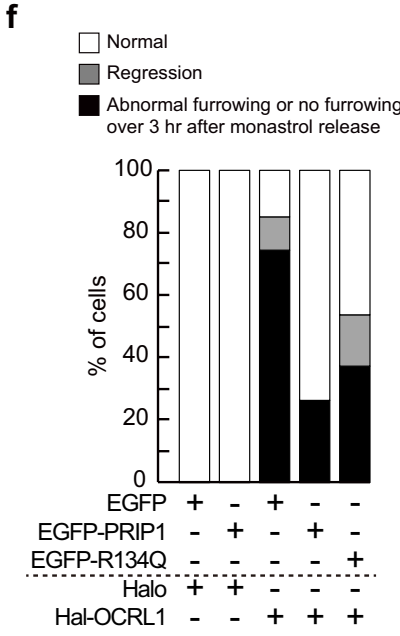

Figure 6. PRIP maintains $\mathrm{PI}(4,5) \mathrm{P}_{2}$ at the cleavage furrow and appropriately manages $\mathrm{PI}(4,5) \mathrm{P}_{2}$-dependent cytokinesis progression. (a-c) A time-lapse experiment using stably EGFP-PLC 8 PH-expressing HEK293 cells and fluorescence recovery after photobleaching (FRAP) analysis. The bleached area (or the pre-bleached area in 0-8 min panels) and non-bleached furrow region are outlined by a white square and a white dotted square on a set of representative images in (a), respectively. Representative images of PRIP2-si1-treated cells are shown as two data sets, a delayed cytokinesis phenotype (middle panel set) and a slightly delayed cytokinesis phenotype (bottom panel set) compared with conventional cytokinesis (control, upper panel set). The graphs in $(\mathbf{b}, \mathbf{c})$ present the relative fluorescence intensity of EGFP-PLC $8 \mathrm{PH}$ [normalised to the value at time 0 (b) or the pre-bleach value (c)] on the non-bleached side of the furrow (b) or bleached cleavage furrow (c) at each time point. The data are presented as the means \pm SD $(\mathrm{n}>20$ for each group). $* * p<0.01$, ***p $p<0.001$ versus the control value at each time point (Kruskal-Wallis test followed by Dunn's multiple comparison test). (d,e) HEK293 cells stably expressing EGFP-tagged PLC $\delta$ PH were transfected with indicated constructs. 
Representative images at a stage of cytokinesis are shown in (d). Graph in (e) shows the EGFP fluorescence intensity (arbitrary units) at the cleavage furrow. The data are presented as the mean \pm SD ( $\mathrm{n}>45$ for each group]. $* * * p<0.001$ (Kruskal-Wallis test followed by Dunn's multiple comparison test). (f) MCF-7 cells stably expressing control vector (EGFP), EGFP-tagged PRIP, or EGFP-tagged PRIP1(R134Q) were cotransfected with Halo-tagged OCRL1 or Halo-tag vector. Quantitative analysis of cytokinetic abnormalities in OCRL1-transfected MCF-7 cells with indicated PRIP1 mutants were evaluated at $3 \mathrm{~h}$ after release from monastrol. Each experiment was repeated at least three times ( $\mathrm{n}>30$ for each group).

Halo vector or Halo-OCRL1 was transiently transfected. Approximately $80 \%$ of OCRL1-transfected EGFP vector-expressing MCF-7 cells showed abnormal cytokinesis (Fig. 6f). Importantly, these abnormal phenotypes were reduced in OCRL1-transfected Prip1-expressing cells and were partially restored in OCRL1-transfected Prip1(R134Q)-expressing cells. These results suggest that PRIP protects $\mathrm{PI}(4,5) \mathrm{P}_{2}$ from OCRL1-mediated hydrolysis, which requires the binding of PRIP and PI $(4,5) \mathrm{P}_{2}$ via the PRIP PH domain.

PRIP prevents $\mathrm{PI}(4,5) \mathrm{P}_{2}$ recognition by $\mathrm{PI}(4,5) \mathrm{P}_{2}$-metabolising enzymes OCRL1 and $\mathrm{PLC} \delta 1$, but not by RhoA, in vitro. We next examined whether PRIP affects the PI $(4,5) \mathrm{P}_{2}$ recognition of OCRL1 and $\mathrm{PLC} 81, \mathrm{PI}(4,5) \mathrm{P}_{2}$-metabolising enzymes. An in vitro co-sedimentation assay was performed using liposomes composed of $100 \%$ phosphatidylcholine (PC) or $5 \% \mathrm{PI}(4,5) \mathrm{P}_{2}$ and $95 \% \mathrm{PC}$ following the procedure described in Fig. 7a (for OCRL1) and Supplementary Fig. S6a (for PLC81). The supernatant (Sup1; Fig. 7a for OCRL1, Supplementary Fig. S6a for PLC81) was prepared from the whole-cell lysate of Halo-OCRL1-expressing or PLC 81 -expressing HeLa cells by centrifugation and used in the precipitation assays. Halo-OCRL1 precipitated with liposomes composed of $5 \% \mathrm{PI}(4,5) \mathrm{P}_{2}$ and $95 \%$ PC but did not precipitate with $100 \%$ PC liposomes (Fig. $7 \mathrm{~b}$, two lanes on the far right). Upon the addition of PRIP1 or PRIP2 to the assay tube, the association between Halo-OCRL1 and PI $(4,5) \mathrm{P}_{2}$ was inhibited (Fig. 7b). Similarly, PLC $\delta 1$ precipitated with liposomes composed of $5 \% \mathrm{PI}(4,5) \mathrm{P}_{2}$ and $95 \% \mathrm{PC}$. However, the association of PLC 81 to $\mathrm{PI}(4,5) \mathrm{P}_{2}$ was inhibited by the addition of PRIP1 (Supplementary Fig. S6b). These data suggest that PRIP, which lacks PLC activity, prevents the binding of PI $(4,5) \mathrm{P}_{2}$ to its metabolising enzymes such as OCRL1 and PLC $\delta 1$, thus negatively modulating $\mathrm{PI}(4,5) \mathrm{P}_{2}$ metabolism.

RhoA also binds to $\mathrm{PI}(4,5) \mathrm{P}_{2}$ and modulates $\mathrm{PI}(4,5) \mathrm{P}_{2}$-mediated cytokinesis ${ }^{9}$. Therefore, to determine whether PRIP affects the association between RhoA and $\mathrm{PI}(4,5) \mathrm{P}_{2}$, we performed the $\mathrm{PI}(4,5) \mathrm{P}_{2}$-liposome sedimentation assay in the presence of OCRL1, RhoA, and PRIP1 (Fig. 7c). The association between OCRL1 and PI $(4,5) \mathrm{P}_{2}$ was inhibited by a low dose of PRIP. However, the association between RhoA and PI(4,5) $\mathrm{P}_{2}$ was not affected, even at a high dose of PRIP (Fig. 7d). These results suggest that PRIP affects the substrate recognition of OCRL1 but does not affect the association between RhoA and $\mathrm{PI}(4,5) \mathrm{P}_{2}$.

\section{Discussion}

Cytokinesis is a highly ordered process requiring an intricate interplay among cytoskeletal, chromosomal, and cell cycle regulatory pathways. Additional cellular processes, including phosphoinositide metabolism on the plasma membrane, are also important for cytokinesis. $\mathrm{PI}(4,5) \mathrm{P}_{2}$, which is an integral signalling molecule despite being a minor component of cellular membranes, is generated by metabolic enzymes at the cleavage furrow and functions there during cytokinesis ${ }^{35}$. Therefore, adequate $\mathrm{PI}(4,5) \mathrm{P}_{2}$ metabolic regulation is crucial for correct cytokinesis progression. The findings in this study demonstrated that the binding of PRIP to PI $(4,5) \mathrm{P}_{2}$ maintains normal $\mathrm{PI}(4,5) \mathrm{P}_{2}$ levels at the cleavage furrow, which leads to the proper completion of cytokinesis. We thus propose that PRIP is a new candidate for protecting $\mathrm{PI}(4,5) \mathrm{P}_{2}$ from metabolism by associated enzymes during cytokinesis. Elucidation of this novel PRIP-mediated cytokinesis regulation pathway may aid our understanding of fundamental cellular mechanisms.

$\mathrm{PI}(4,5) \mathrm{P}_{2}$ interacts with Rho, regulates Rho GTPase activity at the cleavage furrow, and facilitates the recruitment of contractile ring constituents to the cleavage furrow as a scaffold lipid ${ }^{3,9}$. We previously reported that PRIP negatively regulates $\mathrm{PI}(4,5) \mathrm{P}_{2}$ metabolism in migrating cells ${ }^{27}$. In this study, a deficiency of PRIP2 in HeLa cells was found to cause cytokinesis defects and delay by suppressing the accumulation of PI $(4,5) \mathrm{P}_{2}$ and RhoA at the cleavage furrow. The demonstrated PRIP role here is that PRIP maintains $\mathrm{PI}(4,5) \mathrm{P}_{2}$ levels by protecting $\mathrm{PI}(4,5) \mathrm{P}_{2}$ from its metabolising enzymes at the cleavage furrow and regulates the RhoA/pMRLC-dependent progression of cytokinesis. Rho also stimulates PIP5K activity ${ }^{36}$ and ROCK-mediated phosphatase and tensin homologue (PTEN) signalling ${ }^{37}$, which results in the upregulation of $\mathrm{PI}(4,5) \mathrm{P}_{2}$ synthesis. Thus, $\mathrm{PI}(4,5) \mathrm{P}_{2}$, under the guardianship of PRIP, may accelerate the accumulation of RhoA at the cleavage furrow, followed by further $\mathrm{PI}(4,5) \mathrm{P}_{2}$ production by PI5K and/or PTEN. This positive feedback loop contributes to the initiation of the cleavage furrow and the promotion of cleavage furrow ingression to complete cytokinesis.

Anillin, a highly concentrated protein in the cleavage furrow, is a scaffolding protein that links RhoA, F-actin, and myosin II and regulates the contractile ring ${ }^{31}$. RhoA activates ROCK and citron kinase, which phosphorylate the MRLC of non-muscle myosin $\mathrm{II}^{31}$. RhoA also binds and activates formins that elicit actin nucleation and polymerisation. Thus, RhoA coordinates the RhoA/ROCK/pMRLC and RhoA/formin signalling pathways and facilitates actomyosin ring assembly and contraction. In this study, PRIP2-silenced cells exhibited reduced pMRLC levels and the accumulation of F-actin and RhoA at the cleavage furrow. Therefore, these results indicate that PRIP is the most upstream molecule in the RhoA-mediated signalling pathways, profoundly modulating F-actin formation and MRLC phosphorylation. Although PRIP2-silenced cells displayed reduced accumulation of F-actin at the cleavage furrow, the transfection of a phospho-mimic MRLC mutant (AD-MRLC) alone efficiently rescued the cytokinesis delay in PRIP2-silenced cells. It was previously reported that actomyosin contractile ring assembly is regulated by actin-related protein 2 and 3 (ARP2/3), which enhance actin nucleation ${ }^{38,39}$. Myosin II heavy chain accumulates at the cleavage furrow and promotes the elongation of actin bundles ${ }^{29,40}$. These 
a

Whole cell lysate (Halo-OCRL1-expressing HeLa)
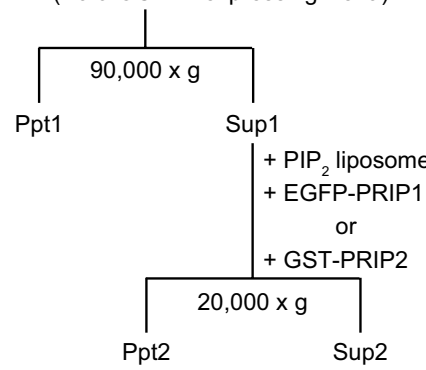

C

Whole cell lysate

(Halo-OCRL1 and Halo-RhoA

-expressing HEK293 cells)

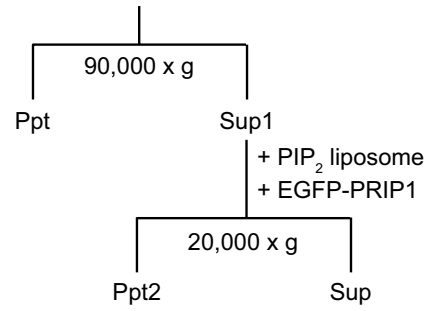

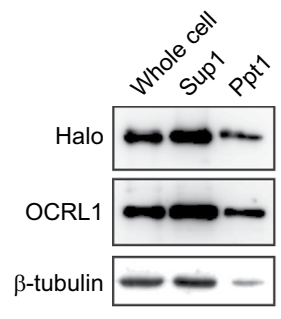

b

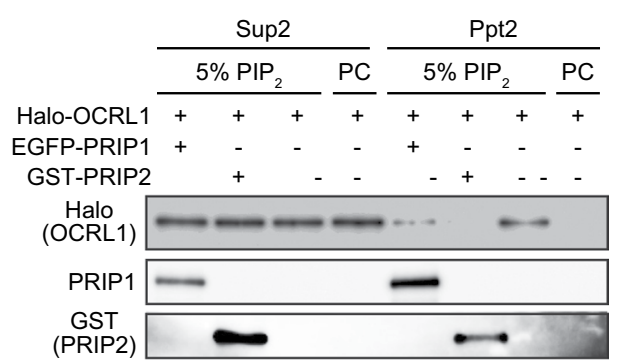

d

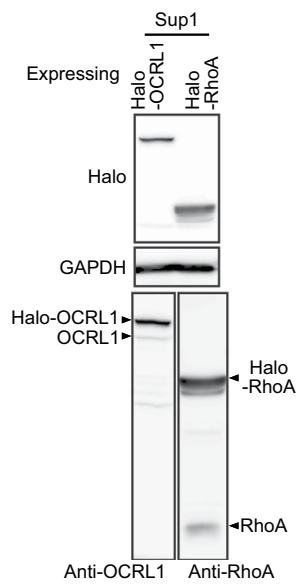

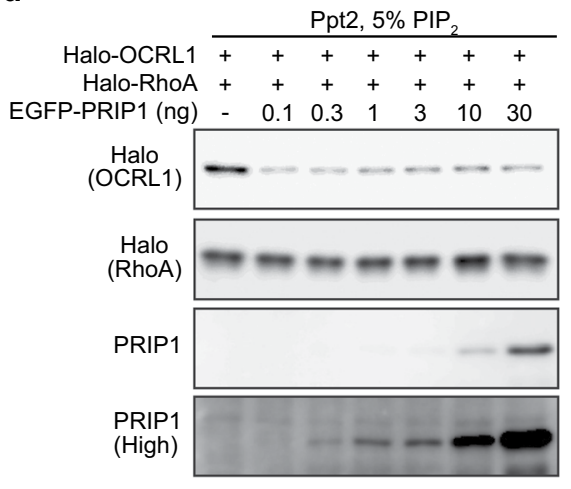

Figure 7. PRIP prevents $\mathrm{PI}(4,5) \mathrm{P}_{2}$ recognition by OCRL1 but not by RhoA. (a-d) $\mathrm{PI}(4,5) \mathrm{P}_{2}$ sedimentation assay was performed in a combination of EGFP-PRIP1, GST-PRIP2, Halo-OCRL1, and Halo-RhoA (a,c). Supernatant 1 (Sup1) contained Halo-OCRL1 (a) or Halo-OCRL1 and Halo-RhoA (c), as confirmed by immunoblotting using indicated antibodies. The fractions of Sup1 in (a or c) precipitated with liposomes [PC, $100 \% \mathrm{PC} ; 5 \% \mathrm{PIP}_{2}, \mathrm{PI}(4,5) \mathrm{P}_{2}: \mathrm{PC}=5: 95$ (molar ratio)] in the presence $(+)$ or absence $(-)$ of recombinant PRIP1 or PRIP2 (b) or with liposomes $\left(5 \% \mathrm{PIP}_{2}\right)$ in the presence of indicated doses of recombinant PRIP1 (d) by centrifugation, respectively. Western blotting of the obtained pellet fraction (Ppt2, b or d) and supernatant (Sup2, b) was performed using indicated antibodies (left side of each panel). Similar data were obtained from three independent experiments, and a set of representative images is shown. The image obtained in a high sensitivity mode (high, d) is shown. Each of the original blots is shown in Supplementary Fig. S8 for (d-f) or Supplementary Fig. S9 for (b).

are independently modulated by the RhoA pathway during cytokinesis. Therefore, regulation of F-actin assembly at basal levels during cytokinesis is most likely modulated by RhoA-independent signalling.

$\mathrm{PI}(4,5) \mathrm{P}_{2}$ is a substrate of PLC, OCRL1, and PI3K. These enzymes are involved in $\mathrm{PI}(4,5) \mathrm{P}_{2}$ turnover. Several PLC isoforms are localised to the cleavage furrow, and PLC activity is required for cytokinesis ${ }^{41,42}$. The overexpression of synaptojanin failed to lead to $\mathrm{PI}(4,5) \mathrm{P}_{2}$ and RhoA accumulation in the cleavage furrow ${ }^{3}$. In addition, a PI(4,5) $\mathrm{P}_{2}$ phosphatase, OCRL, which is mutated in patients with oculocerebrorenal syndrome of Lowe, participates in cell abscission during cytokinesis ${ }^{34}$. Inhibition of the $\mathrm{PI}(4,5) \mathrm{P}_{2}$ kinase PI3K by a PI3K inhibitor such as LY294002 or wortmannin induces a delay in cytokinesis when the inhibitor is added at during furrow cleavage formation ${ }^{43}$. These reports indicate that the dynamic regulation of $\mathrm{PI}(4,5) \mathrm{P}_{2}$ metabolism and $\mathrm{PI}(4,5) \mathrm{P}_{2}$ maintenance at the cleavage furrow are crucial for correct cytokinesis progression. Here, we showed that the overexpression of OCRL1 resulted in reduced $\mathrm{PI}(4,5) \mathrm{P}_{2}$ accumulation at the cleavage furrow and abnormal and delayed cytokinesis. This phenotype was rescued by the additional expression of PRIP1 but not of a $\mathrm{PI}(4,5) \mathrm{P}_{2}$-unbound PRIP mutant. Importantly, FRAP analysis confirmed the inhibitory effect of PRIP on PI $(4,5) \mathrm{P}_{2}$ metabolism at the cleavage furrow. Collectively, our data demonstrate that PRIP protects $\mathrm{PI}(4,5) \mathrm{P}_{2}$ via the PRIP $\mathrm{PH}$ domain from metabolism by its enzymes and maintains sufficient levels of $\mathrm{PI}(4,5) \mathrm{P}_{2}$ for cytokinesis.

RhoA has a carboxy-terminal polybasic tail, which can, through electrostatic interactions, directly bind to several phosphatidylinositols, including $\mathrm{PI}(4,5) \mathrm{P}_{2}$, in vitro. This binding activity requires the recruitment or stabilisation of RhoA at the cleavage furrow ${ }^{9,10,44}$. In our experiments, PRIP competitively inhibited substrate recognition by OCRL1 or PLC $\delta 1$ but did not affect the binding of RhoA to $\mathrm{PI}(4,5) \mathrm{P}_{2}$. These data indicate that the binding mode of RhoA to $\mathrm{PI}(4,5) \mathrm{P}_{2}$ differs from those of PRIP, OCRL1, and PLC $\delta 1$. These results support the positive role of $\mathrm{PRIP}$ in regulating the $\mathrm{PI}(4,5) \mathrm{P}_{2} /$ RhoA signalling pathway. 
PRIP contains an X-Y domain, which is similar to the PLC catalytic core subunit in PLC $\delta 1$. Although PRIP1 and PRIP2 do not have activity, the X-Y domain of PRIP can associate with PI(4,5) $\mathrm{P}_{2}{ }^{15,27}$. PRIP1(R134Q), a $\mathrm{PI}(4,5) \mathrm{P}_{2}$-unbound mutant with a mutation in the $\mathrm{PH}$ domain $^{33}$, slightly accumulated at the cleavage furrow and slightly rescued cytokinesis abnormalities observed in OCRL1-overexpressing cells. The binding of the X-Y domain to $\mathrm{PI}(4,5) \mathrm{P}_{2}$ in the PRIP(R134Q) mutant may inhibit the association between $\mathrm{PI}(4,5) \mathrm{P}_{2}$ and OCRL1. Consequently, the PRIP(R134Q) mutant partially restored cytokinesis.

This is the first study to elucidate the existence of a rheostat for $\mathrm{PI}(4,5) \mathrm{P}_{2}$ metabolism in the cleavage furrow during cytokinesis. Our findings conclusively demonstrate that, even if $\mathrm{PI}(4,5) \mathrm{P}_{2}$ is exposed to a number of its metabolic enzymes, PRIP can maintain the high levels of $\mathrm{PI}(4,5) \mathrm{P}_{2}$ required at the cleavage furrow to promote correct cytokinesis. The continuous phosphoinositide cycling required at the cleavage furrow is regulated by a positive feedback mechanism involving $\mathrm{PI}(4,5) \mathrm{P}_{2}$ accumulation via PRIP-mediated RhoA signalling, which is necessary for normal cytokinesis progression.

\section{Methods}

Plasmids and siRNAs. EGFP-tagged PLC $\delta P H$, Prip1, Prip1(R134Q), and PRIP2; DsRed2-tagged Prip1 and Prip1(R134Q); GST-tagged PRIP2 and PLC 1 ; and Halo-PRIP1 were described previously ${ }^{17,23,45}$. EGFPtagged $A D-M R L C$ and $A A-M R L C$ were described previously ${ }^{46}$. Halo-tagged OCRL1 (FHC01740) and RHOA (FHC01522) were purchased from Promega (Madison, WI, USA). Human PRIP2-siRNAs (si1, 2023-007 and 2023-008; si2, 2023-009 and 2023-010) were purchased from Sigma Genosys (Woodlands, TX, USA). A negative control siRNA (S10C-0600) was purchased from Cosmo Bio (Tokyo, Japan).

Antibodies. Anti-phospho-MRLC (Ser19; \#3671), anti-GAPDH (\#2118), anti-OCRL1 (\#8797), HRP-conjugated anti-rabbit IgG (\#7074), and HRP-conjugated anti-mouse IgG (\#7076) antibodies were purchased from Cell Signaling Technology (Beverly, MA, USA). Anti-PRIP1 and anti-PRIP2 polyclonal antibodies were developed previously ${ }^{47-49}$. Anti- $\beta$-actin antibody (IMG-5142A) was obtained from Imgenex (San Diego, CA, USA). Anti- $\beta$-tubulin (D66) and anti-total MRLC (MY-21) antibodies were purchased from Sigma-Aldrich (St. Louis, MO, USA). Anti-GST antibody (M209-3) was obtained from Medical \& Biological Laboratories (Nagoya, Japan). Anti-RhoA (sc-418) and anti-PLC 1 (D-7) antibodies were purchased from Santa Cruz Biotechnology (Dallas, TX, USA). Anti-HaloTag antibody (G928A) was purchased from Promega. Alexa Fluor 488-conjugated antibodies [anti-rabbit IgG (A11008), anti-mouse IgG (A11001), and anti-mouse IgM (A21042)], Alexa Fluor 594-conjugated anti-mouse IgG (A11005), and Alexa Fluor 405-conjugated antibodies [anti-rabbit IgG (A31556) and anti-mouse IgG (A31553)] were obtained from Invitrogen (Carlsbad, CA, USA). Alexa 594-conjugated anti-mouse IgM (21044) antibody was purchased from Thermo Fisher Scientific (Rockford, IL, USA).

Cell culture and transfection. MCF-7 cells stably expressing EGFP-PRIP1 or EGFP-PRIP1(R134Q) were developed previously ${ }^{27}$. HEK293 cells were transfected with an expression vector encoding EGFP-PLC $\delta \mathrm{PH}$ or a control EGFP vector and cultured in the presence of $1 \mathrm{mg} / \mathrm{ml} \mathrm{G} 418$ (Nakalai Tesque, Kyoto, Japan) for 14 days. Stably EGFP-expressing colonies were selected. Cells were cultured under conventional growth conditions and transfected with plasmids or siRNA using the Lonza 4D-Nucleofector X Unit (Lonza, Basel, Switzerland) or Lipofectamine 3000 (Invitrogen).

Reverse transcription-PCR analysis. A 5- $\mu$ g sample of total RNA from HeLa cells, isolated using an RNeasy minikit (Qiagen, Hilden, Germany), was used for reverse transcription reactions using a cDNA synthesis kit (Takara, Shiga, Japan). PRIP1, PRIP2, and GAPDH were amplified using specific primer pairs whose sequences were previously described ${ }^{27}$. The PCR products were distinguished by agarose gel electrophoresis.

Analyses of living cells and FRAP. Cells were seeded on $\mu$-dishes (Ibidi, Martinsried, Germany) and cultured until attached. Transfection was performed as described above. Cells were synchronised with $50 \mu \mathrm{M}$ monastrol (Cayman Chemical, Ann Arbor, MI, USA) for $12 \mathrm{~h}$, and then monastrol was washed out. For detection of HaloTag, cells were incubated with $1 \mu \mathrm{M}$ of HaloTag TMR ligand or HaloTag Coumarin ligand (Promega) for $1 \mathrm{~h}$ before washout of the ligand. The cells were recorded every minute for $3 \mathrm{~h}$ by live-cell imaging on a BZ-9000 microscope (Keyence, Osaka, Japan). During FRAP analysis, EGFP images were scanned before and after bleaching at low laser power on a confocal laser scanning microscope (Fluoview FV10i; Olympus, Tokyo, Japan). To destroy EGFP fluorescence, maximal laser power was applied to a furrowing region for $30 \mathrm{~s}$. Images were taken before and after bleaching at intervals of $1 \mathrm{~min}$ until completion of cytokinesis. Cytokinesis analysis and measurement of fluorescent intensity were performed using ImageJ $1.45 \mathrm{~s}$ (National Institutes of Health, Bethesda, MD, USA) following previously described methods ${ }^{29}$.

Immunofluorescence and western blotting. Immunofluorescence and western blotting were carried out following previously described methods ${ }^{50,51}$. F-actin was visualised by ActinRed ${ }^{\mathrm{TM}} 555$ ReadyProbes (R37112) (Thermo Fisher Scientific) or Alexa Fluor 350-labelled phalloidin (Invitrogen). RhoA staining was carried out following the previously described method $^{52}$. Fluoview FV10i was used for observations. The acquired images were analysed by Image $1.45 \mathrm{~s}$.

Liposome sedimentation assay. A binding assay of OCRL1 and RhoA to liposomes containing PI $(4,5) \mathrm{P}_{2}$ was carried out using the method described previously ${ }^{27}$, with modifications, using $\mathrm{PI}(4,5) \mathrm{P}_{2}$ (Cayman Chemical) and/or phosphatidylcholine (PC; Sigma-Aldrich). Cell lysates were obtained from cells transiently expressing Halo-OCRL1 and/or Halo-RhoA. Recombinant EGFP-PRIP1 and GST-PRIP2 were purified from MCF-7 cells stably expressing EGFP-Prip1 and HEK293 cells transiently expressed GST-PRIP2. The detailed method is described in Fig. 7a,c. 
Statistical analysis. GraphPad Prism (GraphPad Software, La Jolla, CA, USA) was used for statistical analyses. The non-parametric Kruskal-Wallis test followed by Dunn's multiple comparison test was used. A $p$-value less than 0.05 was considered statistically significant.

\section{Data Availability}

All data generated or analysed during this study are included in this published article and its Supplementary Information file. Other relevant information is available from the authors on reasonable request.

\section{References}

1. Brill, J. A., Wong, R. \& Wilde, A. Phosphoinositide function in cytokinesis. Curr. Biol. 21, R930-934 (2011).

2. Emoto, K., Inadome, H., Kanaho, Y., Narumiya, S. \& Umeda, M. Local change in phospholipid composition at the cleavage furrow is essential for completion of cytokinesis. J. Biol. Chem. 280, 37901-37907 (2005).

3. Abe, M. et al. A role for sphingomyelin-rich lipid domains in the accumulation of phosphatidylinositol-4,5-bisphosphate to the cleavage furrow during cytokinesis. Mol. Cell. Biol. 32, 1396-1407 (2012).

4. Kolay, S., Basu, U. \& Raghu, P. Control of diverse subcellular processes by a single multi-functional lipid phosphatidylinositol 4,5-bisphosphate $\left[\mathrm{PI}(4,5) \mathrm{P}_{2}\right]$. Biochem. J. 473, 1681-1692 (2016).

5. Field, S. J. et al. PtdIns $(4,5) \mathrm{P}_{2}$ functions at the cleavage furrow during cytokinesis. Curr. Biol. 15, 1407-1412 (2005).

6. Echard, A. Phosphoinositides and cytokinesis: the "PIP" of the iceberg. Cytoskeleton 69, 893-912 (2012).

7. Yuce, O., Piekny, A. \& Glotzer, M. An ECT2-centralspindlin complex regulates the localization and function of RhoA. J. Cell Biol. 170, 571-582 (2005).

8. Logan, M. R. \& Mandato, C. A. Regulation of the actin cytoskeleton by PIP in cytokinesis. Biol. Cell 98, 377-388 (2006).

9. Yoshida, S., Bartolini, S. \& Pellman, D. Mechanisms for concentrating Rhol during cytokinesis. Genes Dev. 23, 810-823 (2009).

10. Heo, W. D. et al. $\mathrm{PI}(3,4,5) \mathrm{P}_{3}$ and $\mathrm{PI}(4,5) \mathrm{P}_{2}$ lipids target proteins with polybasic clusters to the plasma membrane. Science 314 , 1458-1461 (2006).

11. Su, K. C., Takaki, T. \& Petronczki, M. Targeting of the RhoGEF Ect2 to the equatorial membrane controls cleavage furrow formation during cytokinesis. Dev. cell 21, 1104-1115 (2011).

12. Kosako, H. et al. Specific accumulation of Rho-associated kinase at the cleavage furrow during cytokinesis: cleavage furrow-specific phosphorylation of intermediate filaments. Oncogene 18, 2783-2788 (1999).

13. Robinson, D. N. \& Spudich, J. A. Towards a molecular understanding of cytokinesis. Trends Cell Biol. 10, 228-237 (2000).

14. Glotzer, M. Animal cell cytokinesis. Annu. Rev. Cell Dev. Biol. 17, 351-386 (2001).

15. Kanematsu, T. et al. Domain organization of p130, PLC-related catalytically inactive protein, and structural basis for the lack of enzyme activity. Eur. J. Biochem. 267, 2731-2737 (2000).

16. Kanematsu, T. et al. A new inositol 1,4,5-trisphosphate binding protein similar to phospholipase C- $\delta_{1}$. Biochem. J. 313, 319-325 (1996).

17. Yagisawa, H. et al. Expression and characterization of an inositol 1,4,5-trisphosphate binding domain of phosphatidylinositolspecific phospholipase C- $\delta_{1}$. J. Biol. Chem. 269, 20179-20188 (1994).

18. Uji, A. et al. Molecules interacting with PRIP-2, a novel Ins $(1,4,5) \mathrm{P}_{3}$ binding protein type 2: Comparison with PRIP-1. Life Sci. 72 , 443-453 (2002).

19. Kikuno, R. et al. Prediction of the coding sequences of unidentified human genes. XIV. The complete sequences of 100 new $\mathrm{cDNA}$ clones from brain which code for large proteins in vitro. DNA Res. 6, 197-205 (1999).

20. Kanematsu, T. et al. Putative inositol 1,4,5-trisphosphate binding proteins in rat brain cytosol. J. Biol. Chem. 267, 6518-6525 (1992).

21. Takeuchi, H. et al. Localization of a high-affinity inositol 1,4,5-trisphosphate/inositol 1,4,5,6-tetrakisphosphate binding domain to the pleckstrin homology module of a new $130 \mathrm{kDa}$ protein: characterization of the determinants of structural specificity. Biochem. J. 318, 561-568 (1996).

22. Takeuchi, H. et al. Inhibition of $\mathrm{Ca}^{2+}$ signalling by p130, a phospholipase-C-related catalytically inactive protein: critical role of the p130 pleckstrin homology domain. Biochem. J. 349, 357-368 (2000).

23. Gao, J. et al. Binding of phospholipase C-related but catalytically inactive protein to phosphatidylinositol 4,5-bisphosphate via the PH domain. Cell. Signal. 21, 1180-1186 (2009).

24. Yoshida, M. et al. D-myo-inositol 1,4,5-trisphosphate-binding proteins in rat brain membranes. J. Biochem. 115, 973-980 (1994).

25. Singh, S. M. \& Murray, D. Molecular modeling of the membrane targeting of phospholipase C pleckstrin homology domains. Protein Sci. 12, 1934-1953 (2003).

26. Ferguson, K. M., Lemmon, M. A., Schlessinger, J. \& Sigler, P. B. Structure of the high affinity complex of inositol trisphosphate with a phospholipase C pleckstrin homology domain. Cell 83, 1037-1046 (1995).

27. Asano, S. et al. Suppression of cell migration by phospholipase C-related catalytically inactive protein-dependent modulation of PI3K signalling. Sci. Rep. 7, 5408 (2017).

28. Sellers, J. R. Myosins: a diverse superfamily. Biochim. Biophys. Acta 1496, 3-22 (2000).

29. Asano, S., Hamao, K. \& Hosoya, H. Direct evidence for roles of phosphorylated regulatory light chain of myosin II in furrow ingression during cytokinesis in HeLa cells. Genes Cells 14, 555-568 (2009).

30. Narumiya, S., Tanji, M. \& Ishizaki, T. Rho signaling, ROCK and mDia1, in transformation, metastasis and invasion. Cancer Metastasis Rev. 28, 65-76 (2009).

31. Glotzer, M. The molecular requirements for cytokinesis. Science 307, 1735-1739 (2005).

32. Heissler, S. M. \& Manstein, D. J. Nonmuscle myosin-2: mix and match. Cell. Mol. Life Sci. 70, 1-21 (2013).

33. Zhang, Z. et al. PRIP (phospholipase C-related but catalytically inactive protein) inhibits exocytosis by direct interactions with syntaxin 1 and SNAP-25 through its C2 domain. J. Biol. Chem. 288, 7769-7780 (2013).

34. Dambournet, D. et al. Rab35 GTPase and OCRL phosphatase remodel lipids and F-actin for successful cytokinesis. Nat. Cell Biol. 13, 981-988 (2011).

35. Janetopoulos, C. \& Devreotes, P. Phosphoinositide signaling plays a key role in cytokinesis. J. Cell Biol. 174, 485-490 (2006).

36. Chong, L. D., Traynor-Kaplan, A., Bokoch, G. M. \& Schwartz, M. A. The small GTP-binding protein Rho regulates a phosphatidylinositol 4-phosphate 5-kinase in mammalian cells. Cell 79, 507-513 (1994).

37. Li, Z. et al. Regulation of PTEN by Rho small GTPases. Nat. Cell Biol. 7, 399-404 (2005)

38. Vartiainen, M. K. \& Machesky, L. M. The WASP-Arp2/3 pathway: genetic insights. Curr. Opin. Cell Biol. 16, 174-181 (2004).

39. Kurisu, S. \& Takenawa, T. WASP and WAVE family proteins: friends or foes in cancer invasion? Cancer Sci. 101, 2093-2104 (2010).

40. Tanaka-Takiguchi, Y. et al. The elongation and contraction of actin bundles are induced by double-headed myosins in a motor concentration-dependent manner. J. Mol. Biol. 341, 467-476 (2004).

41. Wong, R., Fabian, L., Forer, A. \& Brill, J. A. Phospholipase C and myosin light chain kinase inhibition define a common step in actin regulation during cytokinesis. BMC Cell Biol. 8, 15 (2007).

42. Naito, Y., Okada, M. \& Yagisawa, H. Phospholipase C isoforms are localized at the cleavage furrow during cytokinesis. J. Biochem. 140, 785-791 (2006). 
43. Saul, D., Fabian, L., Forer, A. \& Brill, J. A. Continuous phosphatidylinositol metabolism is required for cleavage of crane fly spermatocytes. J. Cell Sci. 117, 3887-3896 (2004).

44. Williams, C. L. The polybasic region of Ras and Rho family small GTPases: a regulator of protein interactions and membrane association and a site of nuclear localization signal sequences. Cell. Signal. 15, 1071-1080 (2003).

45. Takeuchi, H., Kanematsu, T., Misumi, Y. \& Hirata, M. Membrane association of a new inositol 1,4,5-trisphosphate binding protein, p130 is not dependent on the pleckstrin homology domain. Chem. Phys. Lipids 98, 35-47 (1999).

46. Iwasaki, T., Murata-Hori, M., Ishitobi, S. \& Hosoya, H. Diphosphorylated MRLC is required for organization of stress fibers in interphase cells and the contractile ring in dividing cells. Cell Struct. Funct. 26, 677-683 (2001).

47. Kanematsu, T. et al. Role of the PLC-related, catalytically inactive protein p130 in $\mathrm{GABA}_{\mathrm{A}}$ receptor function. EMBO J. 21, 1004-1011 (2002).

48. Mizokami, A. et al. Phospholipase C-related inactive protein is involved in trafficking of $\gamma 2$ subunit-containing $\mathrm{GABA}_{\mathrm{A}}$ receptors to the cell surface. J. Neurosci. 27, 1692-1701 (2007).

49. Kanematsu, T. et al. Modulation of $\mathrm{GABA}_{\mathrm{A}}$ receptor phosphorylation and membrane trafficking by phospholipase $\mathrm{C}$-related inactive protein/protein phosphatase 1 and $2 \mathrm{~A}$ signaling complex underlying brain-derived neurotrophic factor-dependent regulation of GABAergic inhibition. J. Biol. Chem. 281, 22180-22189 (2006).

50. Asano, S. et al. Phospholipase C-related catalytically inactive protein (PRIP) controls KIF5B-mediated insulin secretion. Biol. Open 3, 463-474 (2014).

51. Asano, S. et al. Regulation of cell migration by sphingomyelin synthases: sphingomyelin in lipid rafts decreases responsiveness to signaling by the CXCL12/CXCR4 pathway. Mol. Cell. Biol. 32, 3242-3252 (2012).

52. Kamijo, K. et al. Dissecting the role of Rho-mediated signaling in contractile ring formation. Mol. Biol. Cell 17, 43-55 (2006).

\section{Acknowledgements}

This work was supported by grants from JSPS KAKENHI (Grant Numbers 15K20372 and 17K11644 to S.A., 17H01595 and 17K19766 to M.H., 16K11503 to T.K.).

\section{Author Contributions}

S.A. designed the project, performed the experiments, and wrote the manuscript. Y.I. and M.N. performed experiments. Y.Y., K.H., K.K. and M.H. contributed reagents and analysis tools. T.K. conceived and coordinated the study and wrote the paper. All authors read and approved the final manuscript.

\section{Additional Information}

Supplementary information accompanies this paper at https://doi.org/10.1038/s41598-019-49156-3.

Competing Interests: The authors declare no competing interests.

Publisher's note: Springer Nature remains neutral with regard to jurisdictional claims in published maps and institutional affiliations.

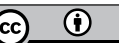

Open Access This article is licensed under a Creative Commons Attribution 4.0 International License, which permits use, sharing, adaptation, distribution and reproduction in any medium or format, as long as you give appropriate credit to the original author(s) and the source, provide a link to the Creative Commons license, and indicate if changes were made. The images or other third party material in this article are included in the article's Creative Commons license, unless indicated otherwise in a credit line to the material. If material is not included in the article's Creative Commons license and your intended use is not permitted by statutory regulation or exceeds the permitted use, you will need to obtain permission directly from the copyright holder. To view a copy of this license, visit http://creativecommons.org/licenses/by/4.0/.

(C) The Author(s) 2019 